\title{
Competitive endogenous RNA (ceRNA) regulation network of IncRNAs, miRNAs, and mRNAs in Wilms tumour
}

\author{
Fucai Tang ${ }^{1 \dagger}$, Zechao $\mathrm{Lu}^{2 \dagger}$, Jiamin Wang ${ }^{3 \dagger}$, Zhibiao $\mathrm{Li}^{4}$, Weijia Wu${ }^{1}$, Haifeng Duan ${ }^{3}$ and Zhaohui He ${ }^{1 *}$ (D)
}

\begin{abstract}
Background: Competitive endogenous RNAs (ceRNAs) have revealed a new mechanism of interaction between RNAs. However, an understanding of the ceRNA regulatory network in Wilms tumour (WT) remains limited.

Methods: The expression profiles of mRNAs, miRNAs and IncRNAs in Wilms tumour samples and normal samples were obtained from the Therapeutically Applicable Research to Generate Effective Treatment (TARGET) database. The EdgeR package was employed to identify differentially expressed IncRNAs, miRNAs and mRNAs. Functional enrichment analyses via the ClusterProfile R package were performed, and the IncRNA-miRNA-mRNA interaction ceRNA network was established in Cytoscape. Subsequently, the correlation between the ceRNA network and overall survival was analysed.

Results: A total of 2037 IncRNAs, 154 miRNAs and 3609 mRNAs were identified as differentially expressed RNAs in Wilms tumour. Of those, 205 IncRNAs, 26 miRNAs and 143 mRNAs were included in the ceRNA regulatory network. The results of Gene Ontology (GO) analysis revealed that the differentially expressed genes (DEGs) were mainly enriched in terms related to response to mechanical stimuli, transcription factor complexes, and transcription factor activity (related to RNA polymerase II proximal promoter sequence-specific DNA binding). The results of the Kyoto Encyclopedia of Genes and Genomes (KEGG) pathway analysis showed that the DEGs were mainly enriched in pathways related to the cell cycle. The survival analysis results showed that 16 out of the 205 IncRNAs, 1 out of 26 miRNAs and 5 out of 143 mRNAs were associated with overall survival in Wilms tumour patients $(P<0.05)$.
\end{abstract}

Conclusions: CeRNA networks play an important role in Wilms tumour. This finding might provide effective, novel insights for further understanding the mechanisms underlying Wilms tumour.

Keywords: Wilms tumour, Long noncoding RNA, Prognosis, Competing endogenous RNA network, MicroRNAs

\section{Background}

Wilms tumour (WT) is the most common renal malignant tumour in children. WT patients have a poor prognosis, although the 5-year overall survival rate is constantly improving with the advancement of diseaseassociated therapies [1]. Chemotherapy, surgery and radiation therapy are the main treatment strategies for WT. However, $50 \%$ of children who have a recurrence after these treatments die from this tumour [2,3]. Novel therapeutic treatments targeting specific mechanisms of WT are still lacking.

\footnotetext{
*Correspondence: gzgyhzh@163.com

†Fucai Tang, Zechao Lu and Jiamin Wang contributed equally to this work.

'Department of Urology, The Eighth Affiliated Hospital, Sun Yat-sen

University, Shenzhen 518033, China

Full list of author information is available at the end of the article
}

Previous studies have demonstrated that numerous key long non-coding RNAs (lncRNAs), microRNAs (miRNAs) and mRNAs are closely related to the pathogenesis of WT, such as LINC00473 [4], miR-483-5p [5], miR-195 [4] and HACE1 [6]. However, there are few reports on the development of prognostic biomarkers in WT. If WT patients who were more likely to have a poor prognosis according to these prognosis biomarker results could be identified, clinicians might be able to apply more aggressive and individualized treatment. Therefore, prognostic biomarkers and targeted therapies in WT need to be identified to improve the clinical outcomes.

In the last decade, the complexity of the human genome has been revealed by advanced RNA sequencing analysis [7]. Under such circumstances, competing 
endogenous RNA (ceRNA) analyses have demonstrated that lncRNAs can communicate with common miRNA response elements and miRNAs to construct an intricate interconnected network and ultimately crosstalk with mRNA. The involvement of the ceRNA regulatory network in tumour initiation and progression has been validated in previous studies [8, 9]. However, the specific ceRNA regulatory network (lncRNA-miRNA-mRNA) in WT remains to be elucidated.

In the present study, a ceRNA regulatory network (205 mRNAs, 26 lncRNAs and 143 miRNAs) was constructed to promote the understanding of how lncRNAs sponge miRNA to regulate gene expression in WT. Subsequently, survival analysis and functional analysis were used to promote a new understanding of the role of the ceRNA regulatory network in WT carcinogenesis. The present study might provide insight into the molecular mechanisms that participate in the progression and tumorigenesis of WT.

\section{Methods}

\section{Data collection and preprocessing}

The raw expression data and corresponding clinical follow-up information were retrieved from the Therapeutically Applicable Research to Generate Effective Treatment (TARGET: https://ocg.cancer.gov/programs/ target; date: February 2019) database. The data (of lncRNAs, mRNAs and miRNAs) in the present study are publicly available. Ethics committee approval was not required because the data in the present study were obtained from the TARGET database. Among the data, miRNA expression data were acquired from 138 samples, including 6 normal samples and 132 WT samples. In addition, mRNA and lncRNA expression data were acquired from 132 samples, including 6 normal samples and 126 WT samples. The differentially expressed lncRNAs (DELs), differentially expressed miRNAs (DEMs) and differentially expressed genes (DEGs) between WT and normal samples were determined via the EdgeR package in the $\mathrm{R}$ software (version 3.3.2) [10]. For the cut-off criteria, a $\mid \log _{2} \mathrm{FC}$ (fold change) $\mid>2$ and a false discovery rate (FDR) of $<0.05$ were used. A flow chart of the analysis procedure is shown in Fig. 1.

\section{Prediction of IncRNA-miRNA and miRNA-mRNA interactions}

DEL-DEM-DEG interactions were divided into DELDEM and DEM-DEG pairs. The StarBase database (http://starbase.sysu.edu.cn/) was employed to change the miRNA sequences. MiRcode [11] (http://www.mircode.org/) is an effective online software that provides the interactions between IncRNAs and miRNAs. MiRTarBase [12] (http:// mirtarbase.mbc.nctu.edu.tw), miRDB [13] (http://www. mirdb.org/miRDB/), and TargetScan [14] (http://www.targetscan.org/) are online tools that were used to retrieve and

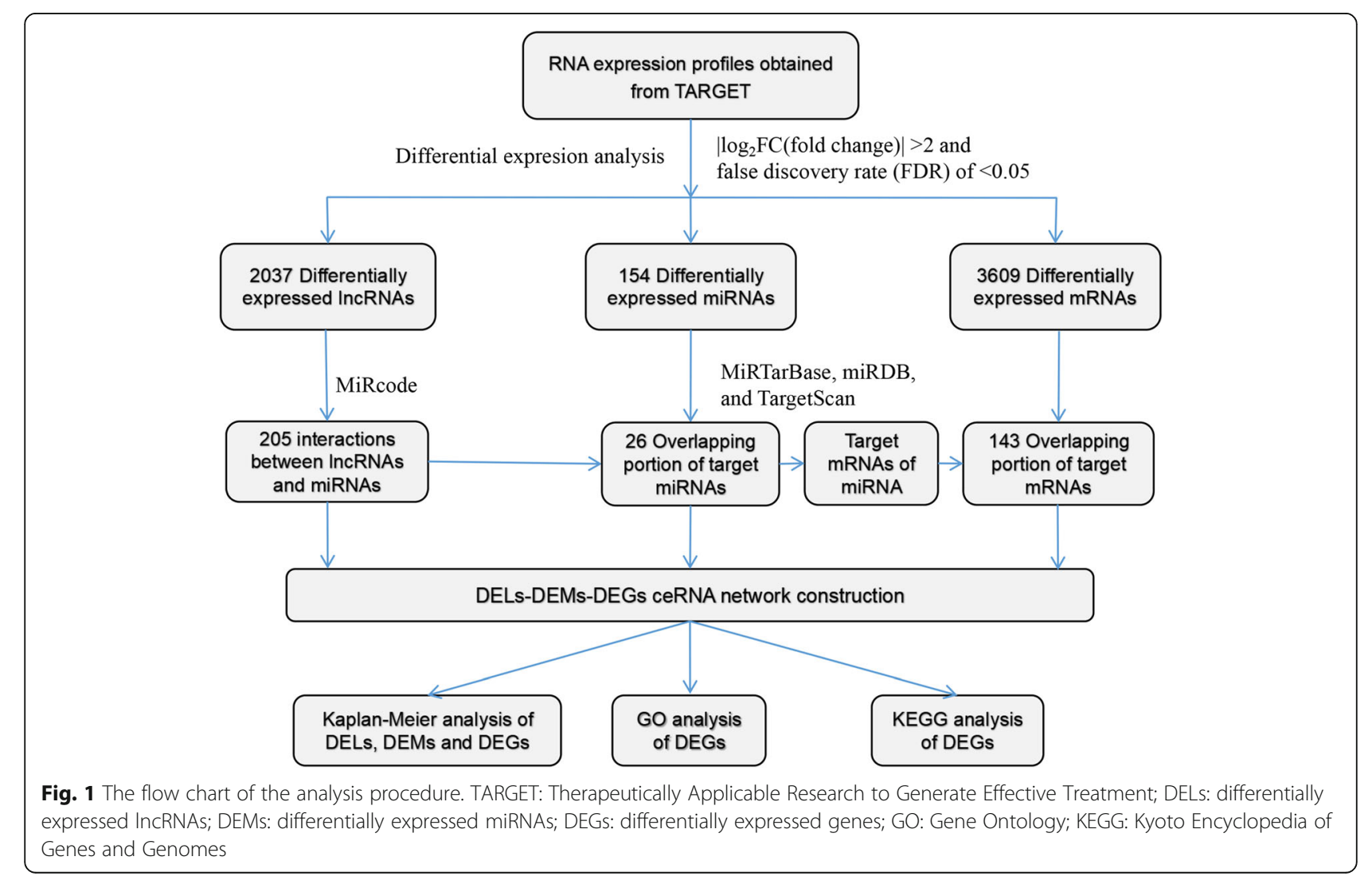




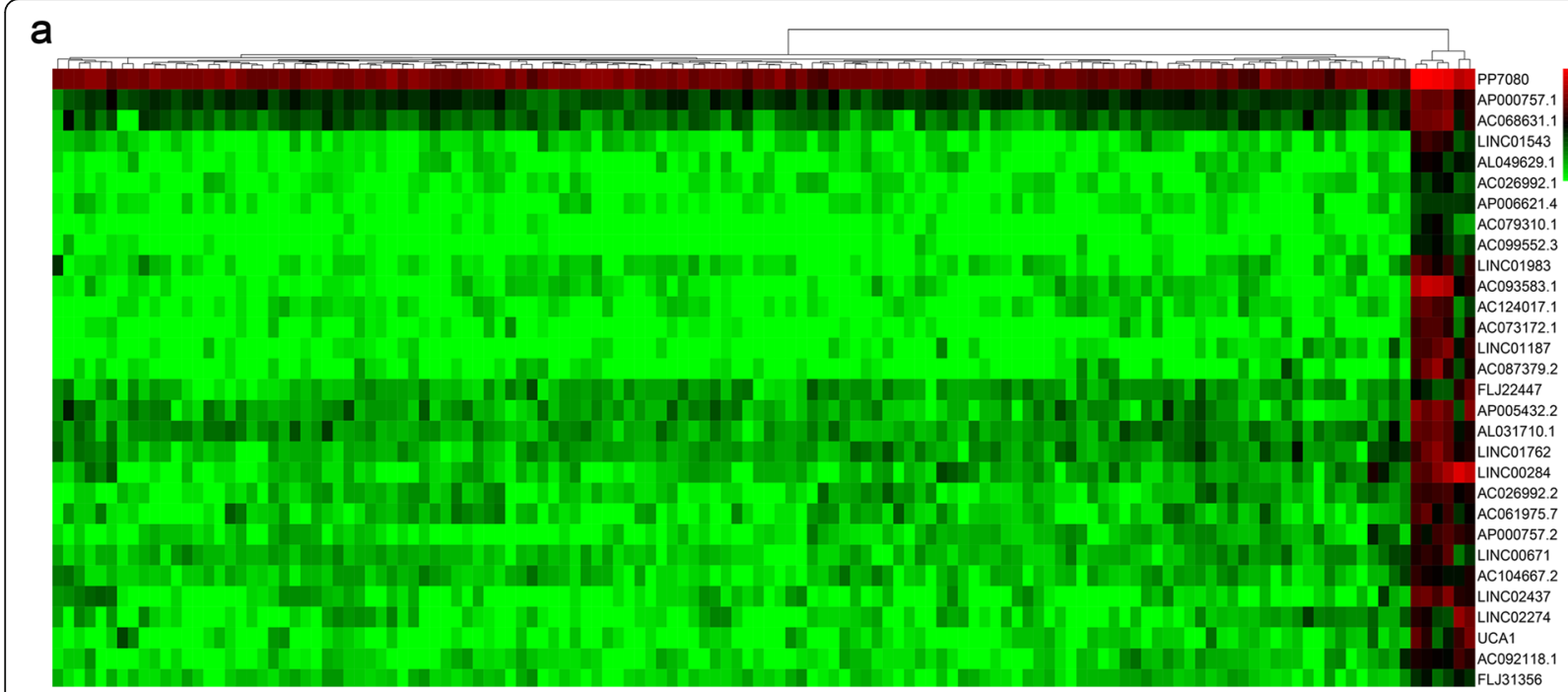

b

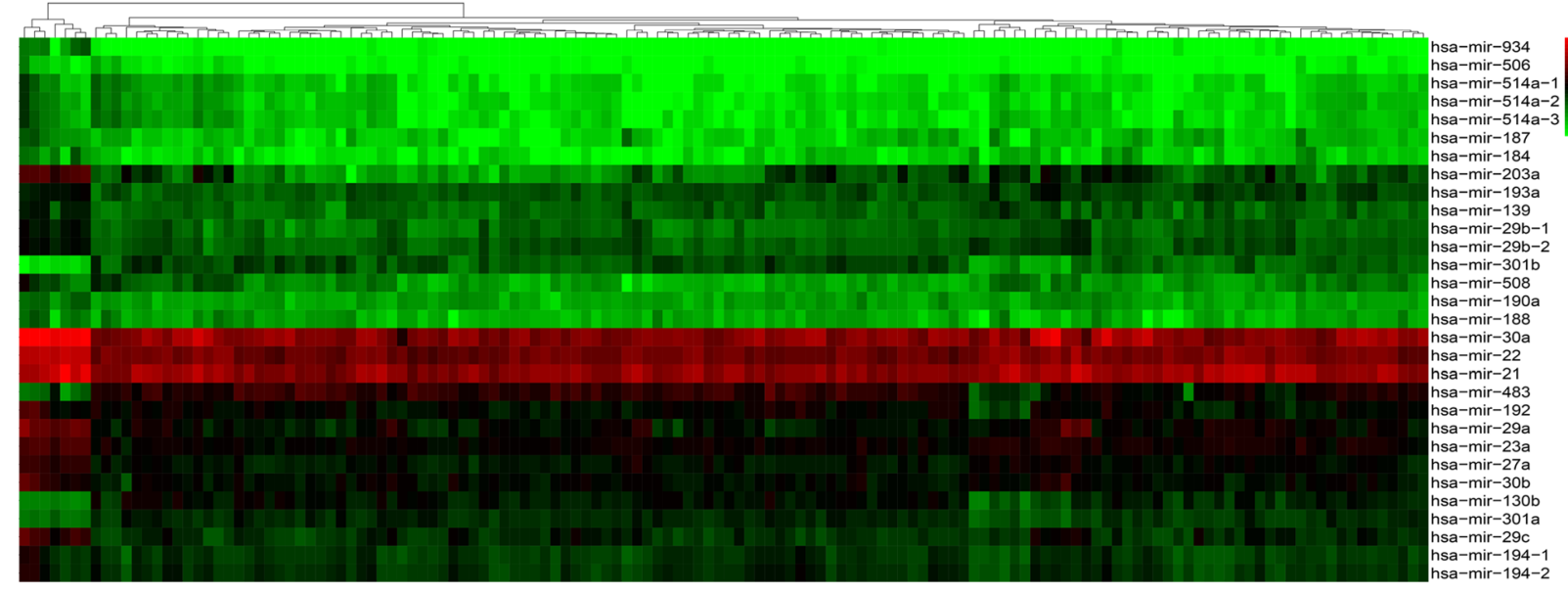

C

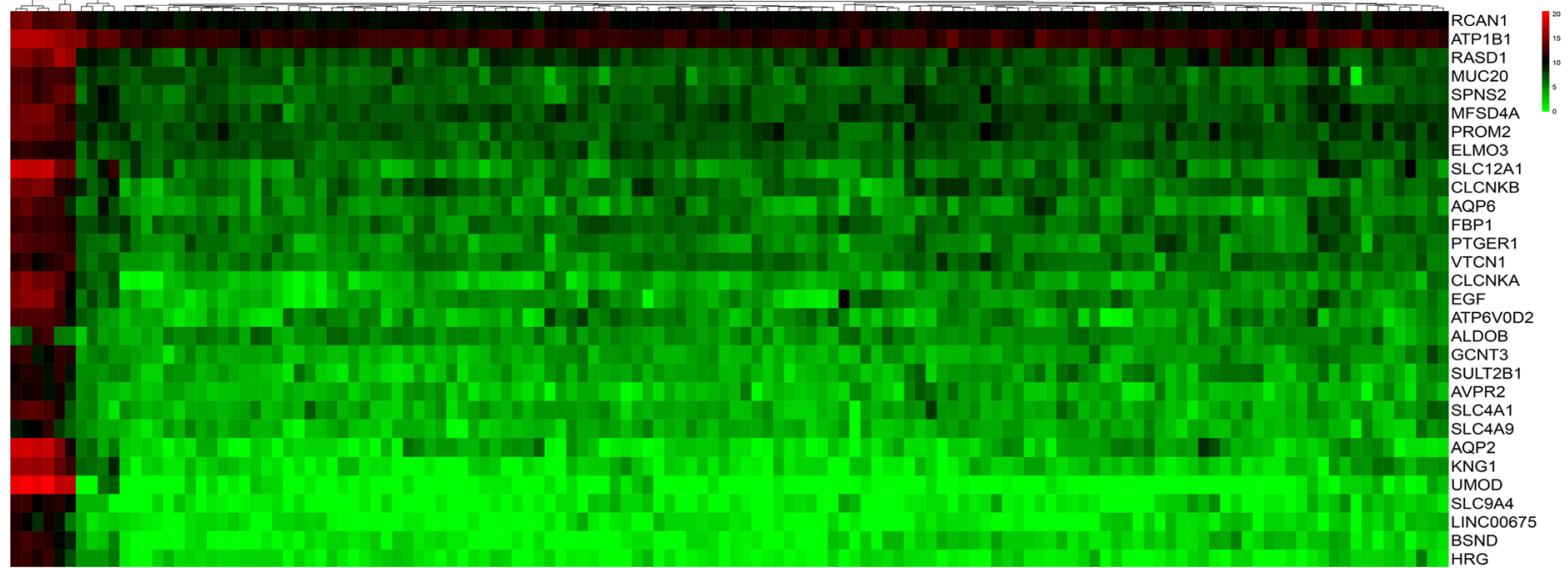

Fig. 2 Heatmap analysis of differentially expressed IncRNAs (a), miRNAs (b), and mRNAs (c) (top 30). Each row represents a sample, and each column represents an IncRNA, miRNA, or mRNA. High or low relative expression is displayed as a red or green strip, respectively 
predict target mRNAs of the miRNAs. A Venn diagram was constructed to obtain the overlapping portion of target miRNAs and mRNAs. Matched DEL-DEM and DEM-DEG interactions were screened for further bioinformatics analysis.

\section{CeRNA network construction and functional enrichment analysis}

Cytoscape software (Version 3.6.1) was utilized to construct and visualize the DEL-DEM-DEG ceRNA network. Cytoscape was used to visualize the molecular interaction networks according to gene expression profiles and annotations. To better comprehend the tumorigenesis mechanisms in WT, Gene Ontology (GO) functional enrichment analysis and Kyoto Encyclopedia of Genes and Genomes (KEGG) pathway enrichment analysis of DEGs in the ceRNA network were performed via the ClusterProfile $R$ package. An FDR < 0.05 was used as a cut-off value.

\section{Survival analysis}

Identification of prognostic DEL, DEM and DEG signatures were executed via the log-rank test and KaplanMeier analysis. The survival curves were constructed

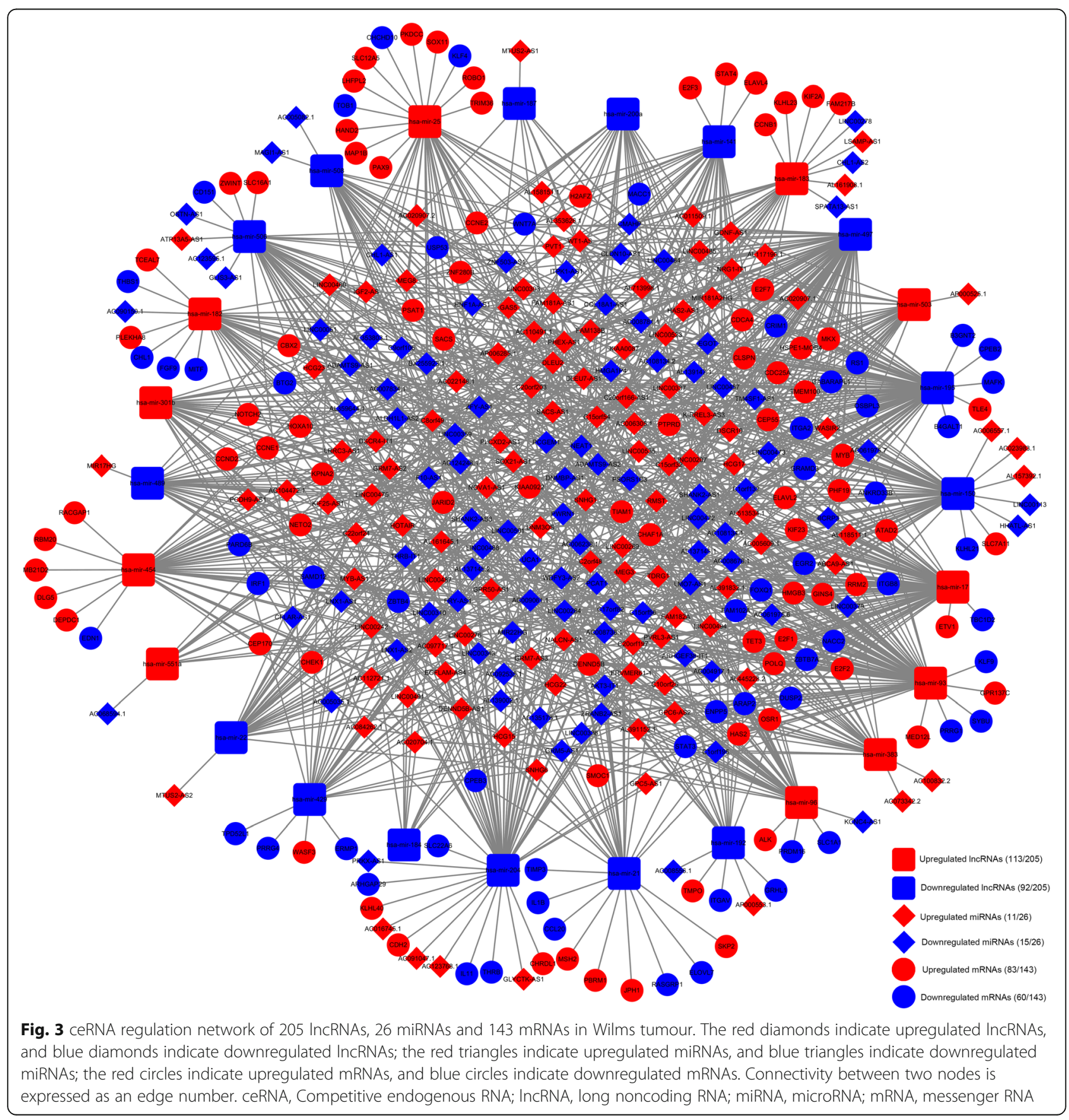


using the 'survival' package. Survival analysis was performed according to Kaplan-Meier univariate survival analysis. $P<0.05$ was selected as the statistical threshold value. All survival analyses were conducted using $\mathrm{R}$ software (version: 3.3.2).

\section{Results}

\section{DEL, DEM and DEG identification in WT}

LncRNA, mRNA, and miRNA expression profiles between WT samples and normal samples acquired from TARGET were analysed in the present study. A total of 2037 DELs, 154 DEMs and 3609 DEGs were identified in the present study. A total of 1247 upregulated and 790 downregulated DELs were identified in WT with a cut-off threshold of a $\mid \log _{2} \mathrm{FC}$ (fold change) $\mid>2$ and a false discovery rate (FDR) of $<0.05$. The distribution of DELs between WT and normal controls is presented as a heatmap plot in Fig. 2a. A total of 105 upregulated and 49 downregulated DEMs were identified in WT. A heatmap plot of the related DEMs between WT and normal controls is shown in Fig. 2b. A total of 1894 upregulated and 1715 downregulated DEGs were identified in WT. The DEG distribution between WT and normal controls is presented as a heatmap plot in Fig. 2c.

\section{CeRNA network construction and functional enrichment analysis}

A dysregulated lncRNA-miRNA-mRNA ceRNA network in WT was constructed according to the interactions of 980 DEL-DEM pairs and 235 DEM-DEG pairs between 205 DELs, 26 DEMs, and 143 DEGs. The ultimate lncRNA-miRNA-mRNA ceRNA regulatory network visualized in Cytoscape is presented in Fig. 3.

GO and KEGG analyses were also performed to reveal the functions of the 143 DEGs that were included in the ceRNA network (see Table 1). The DEGs were enriched in 99 "biological processes (BP)" terms, and the top five terms were response to mechanical stimulus, G1/S transition of mitotic cell cycle, cell cycle G1/S phase transition, muscle cell proliferation, and mesenchymal cell differentiation. The DEGs were enriched in 8 "cellular component (CC)" terms, and the top five terms were transcription factor complex, cyclin-dependent protein kinase holoenzyme complex, nuclear chromatin, nuclear transcription factor complex, and Flemming body. The DEGs were enriched in 10 "molecular function (MF)" terms. The top five terms were transcription factor activity (related to RNA polymerase II proximal promoter sequence-specific DNA binding), transcriptional activator activity (related to RNA polymerase II transcription

Table 1 The enriched GO terms (top 5) and KEGG pathways (top 5) of the DEGs

\begin{tabular}{|c|c|c|c|c|}
\hline Category & Term & ID & Count & FDR \\
\hline GO BP & response to mechanical stimulus & GO:0009612 & 11 & 0.001184756 \\
\hline GO BP & G1/S transition of mitotic cell cycle & GO:0000082 & 12 & 0.001333555 \\
\hline GO BP & cell cycle G1/S phase transition & GO:0044843 & 12 & 0.001784413 \\
\hline GO BP & muscle cell proliferation & GO:0033002 & 10 & 0.002607351 \\
\hline GO BP & mesenchymal cell differentiation & GO:0048762 & 9 & 0.009770918 \\
\hline GO CC & transcription factor complex & GO:0005667 & 10 & 0.01896361 \\
\hline GO CC & cyclin-dependent protein kinase holoenzyme complex & GO:0000307 & 4 & 0.01896361 \\
\hline GO CC & nuclear chromatin & GO:0000790 & 10 & 0.01896361 \\
\hline GO CC & nuclear transcription factor complex & GO:0044798 & 7 & 0.024102204 \\
\hline GO CC & Flemming body & GO:0090543 & 3 & 0.037639391 \\
\hline GO MF & transcription factor activity, RNA polymerase II proximal promoter sequence-specific DNA binding & GO:0000982 & 15 & 0.000130468 \\
\hline GO MF & $\begin{array}{l}\text { transcriptional activator activity, RNA polymerase II transcription regulatory region sequence-specific } \\
\text { DNA binding }\end{array}$ & GO:0001228 & 15 & 0.000130468 \\
\hline GO MF & proximal promoter sequence-specific DNA binding & GO:0000987 & 14 & 0.000902732 \\
\hline GO MF & transcriptional activator activity, RNA polymerase II proximal promoter sequence-specific DNA binding & GO:0001077 & 11 & 0.000902732 \\
\hline GO MF & $\begin{array}{l}\text { transcriptional repressor activity, RNA polymerase II transcription regulatory region sequence-specific } \\
\text { DNA binding }\end{array}$ & GO:0001227 & 10 & 0.000902732 \\
\hline KEGG & Cell cycle & hsa04110 & 10 & $6.52 \mathrm{E}-06$ \\
\hline KEGG & Small cell lung cancer & hsa05222 & 8 & 4.96E-05 \\
\hline KEGG & p53 signaling pathway & hsa04115 & 7 & 7.55E-05 \\
\hline KEGG & MicroRNAs in cancer & hsa05206 & 12 & 0.00013503 \\
\hline KEGG & Cellular senescence & hsa04218 & 9 & 0.000137065 \\
\hline
\end{tabular}

Abbreviations: $F D R$, False discovery rate 
regulatory region sequence-specific DNA binding), proximal promoter sequence-specific DNA binding, transcriptional activator activity (related to RNA polymerase II proximal promoter sequence-specific DNA binding), and transcriptional repressor activity (related to RNA polymerase II transcription regulatory region sequencespecific DNA binding). Additionally, KEGG pathway analysis showed that DEGs were enriched in 30 pathways, such as pathways related to cell cycle, small-cell lung cancer, p53 signalling, microRNAs in cancer, and cellular senescence (Table 1 and Fig. 4).

\section{Survival analysis}

Kaplan-Meier analysis was performed using the combined clinical follow-up information and gene expression profiles of 205 lncRNAs, 26 miRNAs and 143 mRNAs in the ceRNA network in WT samples. According to the analysis, 16 lncRNAs among 205 DELs were closely related to the overall survival of WT patients (Fig. 5). For AC005609.1, AC135178.1, AL391832.1, AL445228.2, ATP13A5-AS1, DENND5BAS1, DLEU2, GRM7-AS3, LINC00303, LINC00473 and LMO7-AS1, low expression was related to a high overall survival rate in WT patients. High expression of AC068594.1, MEG3, NRG1-IT1, RMST and SNHG6 was related to high survival rates in WT patients. In addition, 1 miRNA among 26 DEMs was closely related to the prognosis of WT patients (Fig. 6). High expression of hsa-mir-200a was related to high survival rates in WT patients. Five mRNAs among 143 DEGs were closely related to the overall survival of WT patients (Fig. 7). For CEP55, DEPDC1, PHF19 and TRIM36, low expression was related to a high overall survival rate in WT patients. High expression of KIAA0922 was significantly related to high survival rates in WT patients.

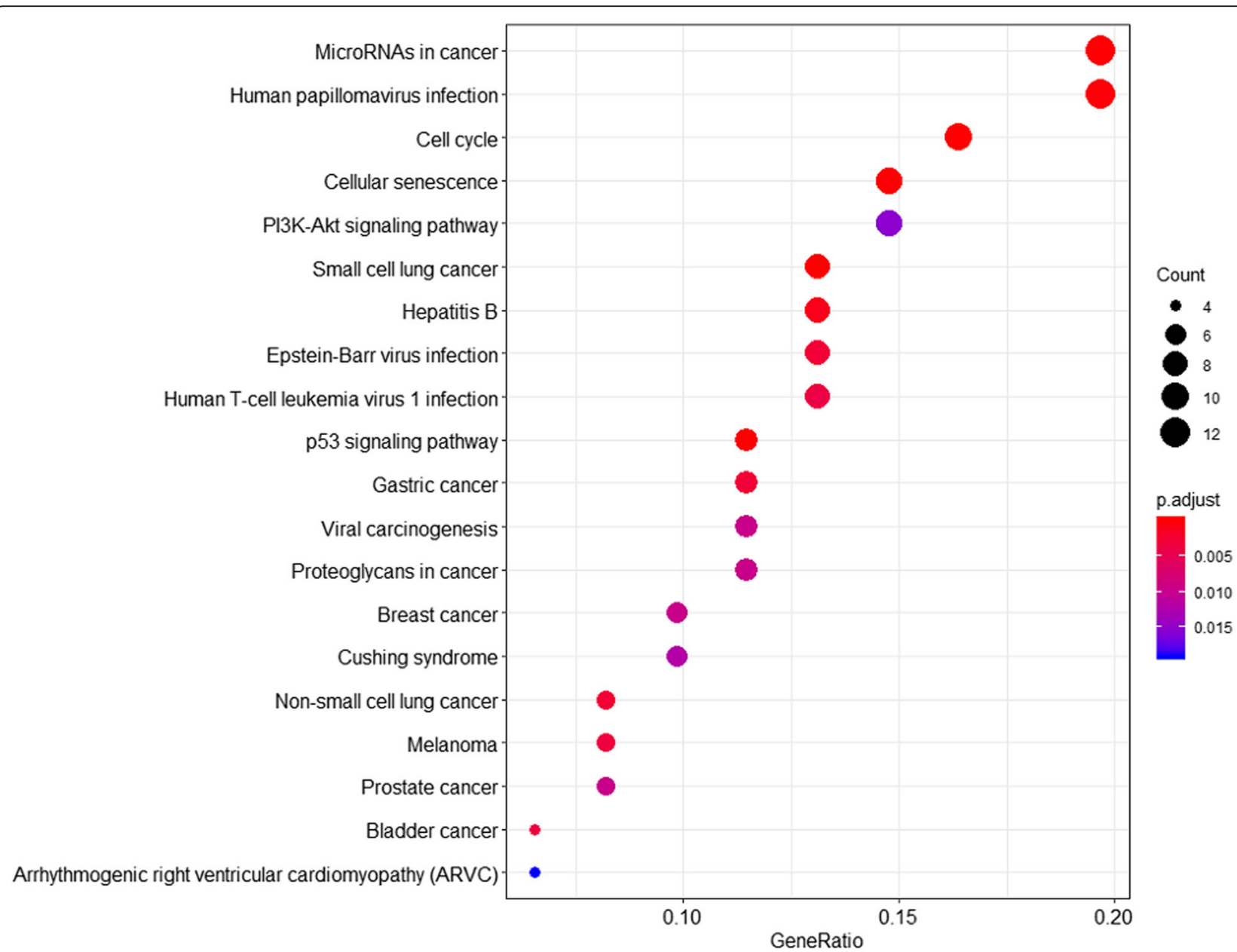

Fig. 4 The KEGG pathways enriched in the genes involved in the ceRNA network of Wilms tumour. The X-axis represents the gene ratio for the indicated genes, with the top 20 KEGG pathways shown on the Y-axis. The graph displays only the top 20 KEGG terms. In this graph, the gradient of blue to red denotes a change in the significance of the correlation from low and high, respectively, and the different sizes of the dots represent related mRNA numbers 


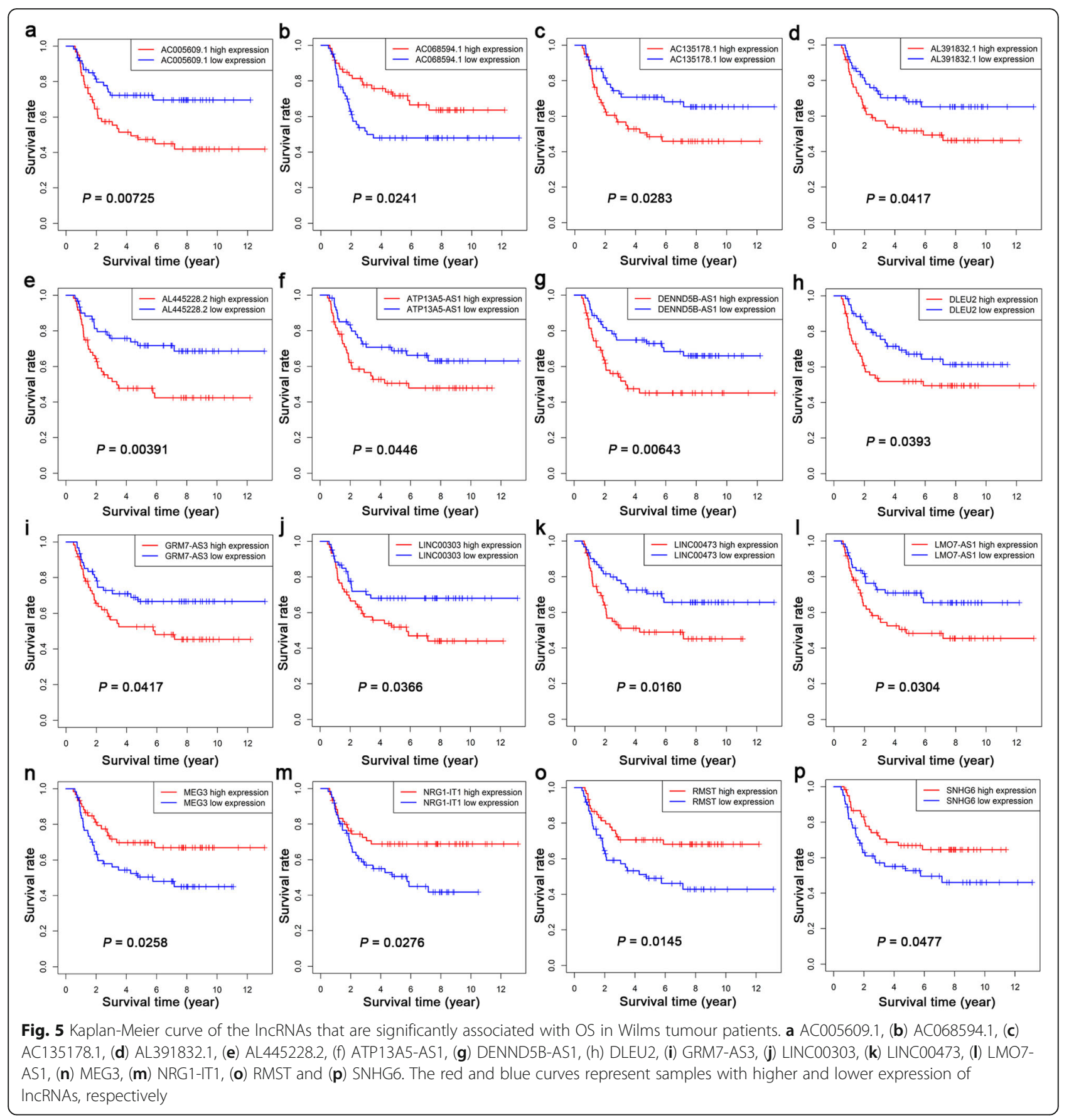

\section{Discussion}

WT is a kind of renal malignant tumour. Although overall survival in WT patients is constantly improved, disease recurrence and poor prognosis are still the main causes of cancer-related death in childhood [1]. Dysregulated genes are considered to be the major cause of tumorigenesis in WT. Recently, increasing attention has been paid to the crucial roles of the ceRNA network in gene expression regulation at the transcription, posttranscription and translation levels. A previous study reported the regulatory role of ceRNA networks in the proliferation, metastasis and invasion of cancer $[15,16]$. To better understand how the ceRNA regulatory network affects WT, large-scale WT data from the TARGET database were analysed, and a dysregulated ceRNA regulatory network in WT was successfully constructed. A dysregulated ceRNA network in WT was constructed according to the interactions of 980 DEL-DEM pairs and 235 DEM-DEG pairs between 205 DELs, 26 DEMs, and 143 DEGs. In addition, Kaplan-Meier curves were 


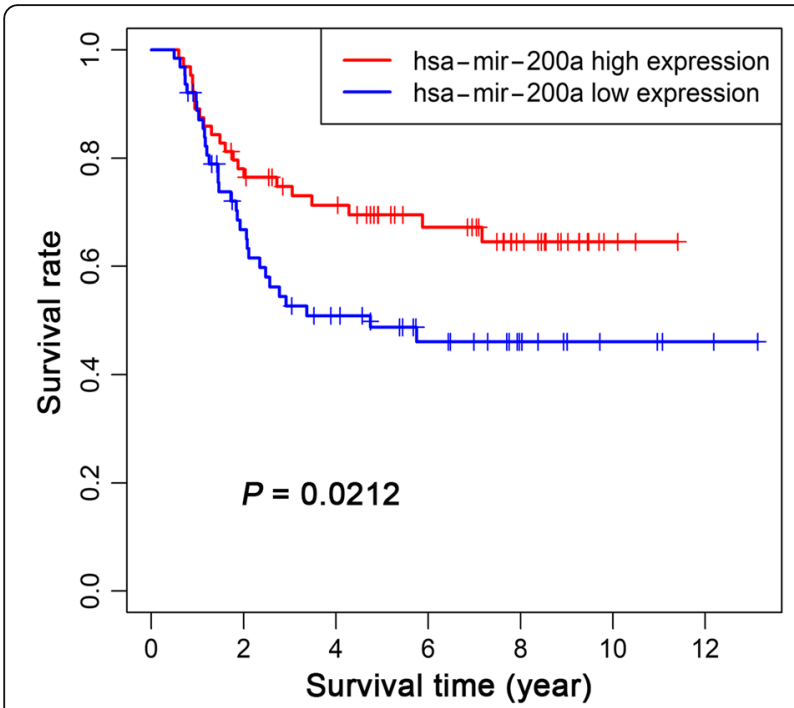

Fig. 6 Kaplan-Meier curve of hsa-mir-200a, which was significantly associated with overall survival in Wilms tumour patients. The red and blue curves represent samples with higher and lower expression of miRNAs, respectively generated to identify prognostic biomarkers in WT. Sixteen differentially expressed lncRNAs (AC005609.1, AC068594.1, AC135178.1, AL391832.1, AL445228.2, ATP13A5-AS1, DENND5B-AS1, DLEU2, GRM7-AS3, LINC00303, LINC00473, LMO7-AS1, MEG3, NRG1IT1, RMST and SNHG6), 1 differentially expressed miRNA (hsa-mir-200a) and 5 differentially expressed mRNAs (CEP55, DEPDC1, KIAA0922, PHF19 and TRIM36) were shown to be significantly associated with the overall survival rate in WT.

Long noncoding RNAs (lncRNAs) are defined as noncoding RNAs longer than 200 nucleotides [17]. IncRNAs were shown to be involved in a variety of biological regulatory functions, including metastasis and tumorigenesis of cancer [18, 19]. A total of 1247 upregulated and 790 downregulated DELs were identified in the present study. A total of 205 DELs were included in the construction of the ceRNA network. Additionally, 16 out of the 205 DELs were associated with overall survival in WT patients $(P<0.05)$. Some differentially expressed lncRNAs in our analysis have been investigated in WT: for example, LINC00473 was show to be capable of
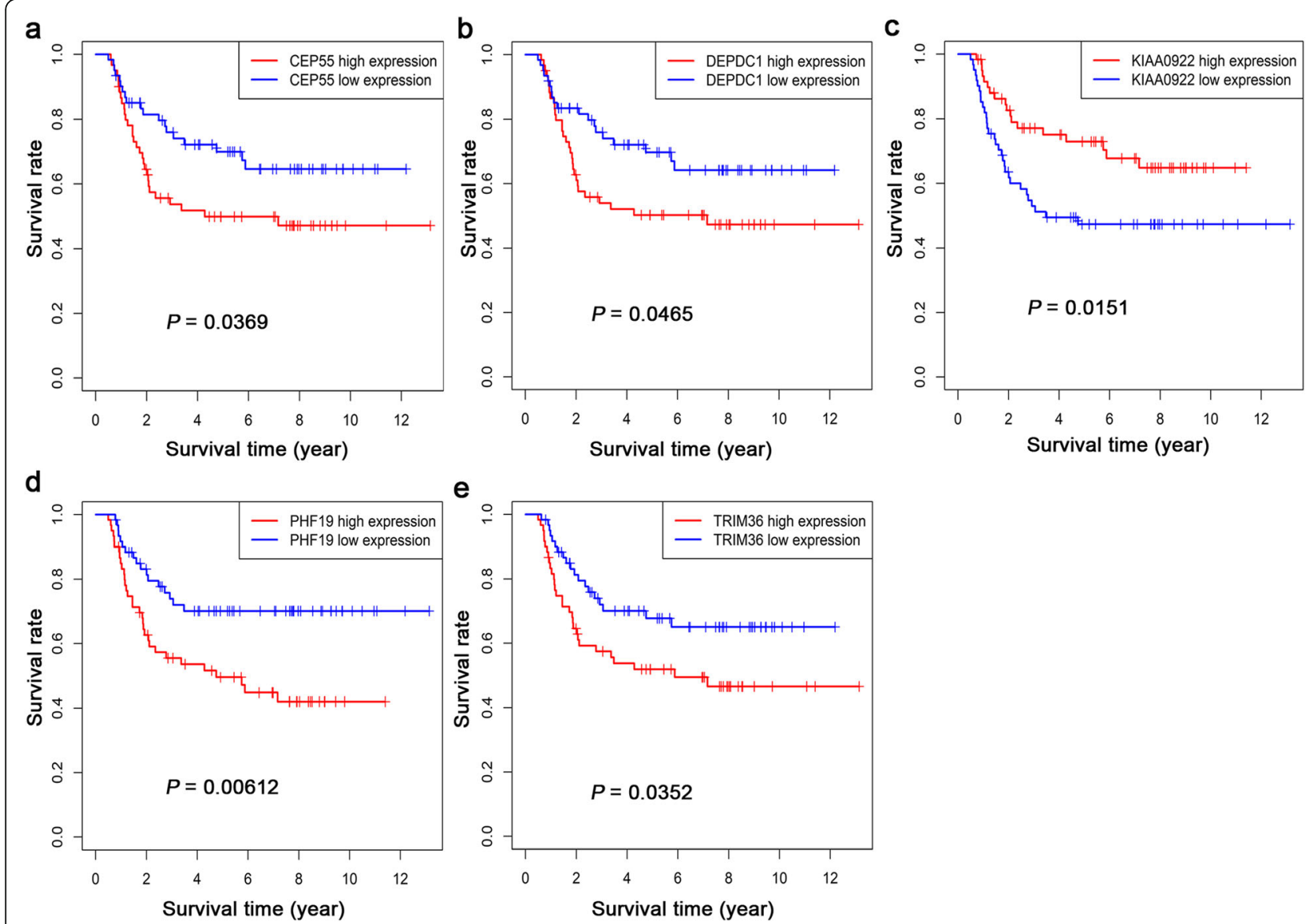

Fig. 7 Kaplan-Meier curve of the mRNAs that are significantly associated with OS in Wilms tumour patients. (a) CEP55, (b) DEPDC1, (c) KIAA0922, (d) PHF19 and (e) TRIM36. The red and blue curves represent samples with higher and lower expression of mRNAs, respectively 
decreasing miR-195 expression levels and inhibiting miR-195 function in WT [4]. A dysregulated lncRNA signature including LINC00473/miR-195/IKKa was shown to play a protumorigenic pathogenesis role in WT [4]. Additionally, SNHG6 was shown to be overexpression in WT tissues, the knockout of SNHG6 can inhibit proliferation, migration and invasion of WT cells, which showed that SNHG6 was an oncogene of WT development [20]. The abovementioned molecular experiments partially supported our results in the present study. The identified lncRNAs might serve as prognostic biomarkers and therapeutic targets in WT. In previous studies, RMST was shown to possibly inhibit cell proliferation, invasion, and migration, enhance cell apoptosis, and regulate the cell cycle to act as a tumour suppressor in triple-negative breast cancer [21]. MEG3, a myeloid-related lncRNA, plays a tumour suppressor role in various solid neoplasms [22, 23]. DLEU2 was shown to control miR-16-1 to regulate proliferation, invasion and migration in laryngeal cancer [24]. Few studies, however, have explored the relationship between the abovementioned DELs and tumorigenesis in WT. Additionally, little is known about the regulatory role of AC005609.1, AC068594.1, AC135178.1, AL391832.1, AL445228.2, ATP13A5-AS1, DENND5BAS1, GRM7-AS3, LINC00303, LMO7-AS1 and NRG1IT1 in cancer. Therefore, further studies are needed to illuminate the molecular and biological mechanisms of these DELs in WT.

MicroRNAs are single-stranded and 18-25 nucleotide-long noncoding RNAs that target regulated gene expression [25]. DEMs in WT, including 105 upregulated and 49 downregulated DEMs, were summarized in the present study. In the present study, the ceRNA network contained 26 differentially expressed miRNAs. However, only one miRNA (miR200a) was related to overall survival in WT patients. miR-200a is an important member of the miR-200 family. It has been reported that miR-200a participates in several biological processes to control the progression of cancer [26, 27]. miR-200a was shown to inhibit the survival, proliferation and invasion of glioma cells by target regulating FOXA1[27]. In addition, miR-200a might inactivate BRD4-mediated AR signalling to inhibit the progression of prostate cancer [26]. Moreover, previous studies have reported that miR-200a participates in the development and occurrence of oesophageal cancer, breast cancer and endometrial cancer by target regulating specific genes [28-30]. However, there is no research to clearly elucidate the role of miR-200a in WT. The understanding of the role of miR-200a in the progression of WT is limited and requires more targeted molecular studies.
To further investigate the related cellular mechanisms in WT, GO and KEGG analyses of 143 DEGs in the ceRNA network were performed. The GO analysis results showed that the DEGs were mainly enriched in terms related to response to mechanical stimuli, transcription factor complexes and transcription factor activity (related to RNA polymerase II proximal promoter sequence-specific DNA binding). The KEGG analysis results indicated that DEGs in the ceRNA network were mainly enriched in pathways related to the cell cycle, small-cell lung cancer, p53 signalling, microRNAs in cancer, and cellular senescence. In recent years, many studies have reported the same findings. The PI3KAKT-p53 signalling pathway is involved in the tumorigenesis of WT and might represent a potential target in the future [31]. MiRNAs, which are single-stranded and 18-25-nucleotide-long noncoding RNAs [25], were involved in regulating proliferation, the cell cycle and apoptosis in WT [32, 33]. Cellular senescence was reported to be responsible for restricted proliferation in WT, and this result was linked to increased p21 expression and was independent of p53 expression [34]. The abovementioned related studies and experiments partially support our GO and KEGG analysis results in the present study.

The major limitation of the present study is that confirmation of the differentially expressed lncRNAs, miRNAs, mRNA and relative pathways in tumour tissues and blood is lacking. Further targeted studies related to this ceRNA network need to be designed to verify and investigate these valuable RNAs in the progression of WT.

\section{Conclusions}

In summary, differentially expressed lncRNAs, mRNAs, and miRNAs were identified, and a functional lncRNAmiRNA-mRNA ceRNA regulatory network for WT tumorigenesis was successfully constructed. Significantly altered lncRNAs, miRNAs and mRNAs might serve as prognostic biomarkers and therapeutic targets for tumorigenesis in WT. The ceRNA regulatory network might illuminate the inner molecular mechanism involved in the progression and tumorigenesis of WT.

\section{Abbreviations \\ ceRNA: competitive endogenous RNA; DEGs: Differentially expressed genes; DELs: Differentially expressed IncRNAs; DEMs: Differentially expressed miRNAs; FDR: False discovery rate; GO: Gene Ontology; KEGG: Kyoto Encyclopedia of Genes and Genomes; IncRNA: Long non-coding RNA; miRNA: microRNA; TARGET: Therapeutically Applicable Research to Generate Effective Treatment; WT: Wilms tumour}

\section{Acknowledgements}

The authors would like to thank Mr. Weipeng Zheng at the Department of Orthopedics, Guangzhou First People's Hospital, Guangzhou Medical University (Guangzhou, China), for his assistance in the use of the $R$ programming language. 


\section{Authors' contributions}

ZH, FT and ZL conceived the study. ZH, FT, ZL, JW and ZL obtained datas and performed statistical analyses. FT, ZL and JW wrote the paper. WW and HD critically read the manuscript. $\mathrm{ZH}$ revised the manuscript. All of the authors read and approved the manuscript.

\section{Funding}

The present study was supported by a research start-up fee for the Eighth Affiliated Hospital, Sun Yat-sen University (grant no. zdbykyqdf005). The funding body played no role in the design of the study and collection, analysis, and interpretation of data and in writing the manuscript.

\section{Availability of data and materials}

The results published here are in whole or part based upon data generated by the Therapeutically Applicable Research to Generate Effective Treatments (https:// ocg.cancer.gov/programs/target) initiative, phs000218. The data used for this analysis are available at https:/portal.gdc.cancer.gov/projects/TARGET-WT.

\section{Ethics approval and consent to participate}

In the present study, the data were download from the Therapeutically Applicable Research to Generate Effective Treatment database; therefore, this article does not contain any studies with human participants or animals performed by any of the authors. Thus, no ethical approval or patient consent were required.

\section{Consent for publication}

Not applicable.

\section{Competing interests}

All authors declare that they have no competing interests.

\section{Author details}

'Department of Urology, The Eighth Affiliated Hospital, Sun Yat-sen University, Shenzhen 518033, China. ${ }^{2}$ First Clinical College of Guangzhou Medical University, Guangzhou 510230, China. ${ }^{3}$ Department of Urology, Minimally Invasive Surgery Center, Guangdong Provincial Key Laboratory of Urology, The First Affiliated Hospital of Guangzhou Medical University, Guangzhou 510230, China. ${ }^{4}$ Three Clinical College of Guangzhou Medical University, Guangzhou 510230, China.

\section{Received: 5 June 2019 Accepted: 8 December 2019} Published online: 16 December 2019

\section{References}

1. Routh JC, Grundy PE, Anderson JR, Retik AB, Kurek KC. B7-h1 as a biomarker for therapy failure in patients with favorable histology Wilms tumor. J Urol. 2013;189:1487-92.

2. Malogolowkin M, Cotton CA, Green DM, Breslow NE, Perlman E, Miser J, et al. Treatment of Wilms tumor relapsing after initial treatment with vincristine, actinomycin D, and doxorubicin. A report from the National Wilms tumor study group. Pediatr Blood Cancer. 2008:50:236-41.

3. Cone EB, Dalton SS, Van Noord M, Tracy ET, Rice HE, Routh JC. Biomarkers for Wilms tumor: a systematic review. J Urol. 2016:196:1530-5.

4. Zhu S, Fu W, Zhang L, Fu K, Hu J, Jia W, et al. LINC00473 antagonizes the tumour suppressor miR-195 to mediate the pathogenesis of Wilms tumour via IKKalpha. Cell Prolif. 2018;51:e12416. https://doi.org/10.1111/cpr.12416.

5. Liu K, He B, Xu J, Li Y, Guo C, Cai Q, et al. miR-483-5p targets MKNK1 to suppress Wilms' tumor cell proliferation and apoptosis in vitro and in vivo. Med Sci Monit. 2019;25:1459-68.

6. Jia W, Deng Z, Zhu J, Fu W, Zhu S, Zhang LY, et al. Association between HACE1 gene polymorphisms and Wilms' tumor risk in a Chinese population. Cancer Investig. 2017;35:633-8.

7. Schwartz MH, Wang H, Pan JN, Clark WC, Cui S, Eckwahl MJ, et al. Microbiome characterization by high-throughput transfer RNA sequencing and modification analysis. Nat Commun. 2018;9:5353.

8. Xu Y, Chen J, Yang Z, Xu L. Identification of RNA expression profiles in thyroid cancer to construct a competing endogenous RNA (ceRNA) network of mRNAs, long noncoding RNAs (InCRNAs), and microRNAs (miRNAs). Med Sci Monit. 2019;25:1140-54

9. Liu Q, Deng J, Wei X, Yuan W, Ma J. Integrated analysis of competing endogenous RNA networks revealing five prognostic biomarkers associated with colorectal cancer. J Cell Biochem. 2019;120(7):11256. https://doi.org/10.1002/jcb.28403.
10. Gong Y, Zou B, Chen J, Ding L, Li P, Chen J, et al. Potential Five-MicroRNA Signature Model for the Prediction of Prognosis in Patients with Wilms Tumor. Med Sci Monit. 2019;25:5435-44.

11. Jeggari A, Marks DS, Larsson E. miRcode: a map of putative microRNA target sites in the long non-coding transcriptome. Bioinformatics. 2012;28:2062-3.

12. Chou CH, Shrestha S, Yang CD, Chang NW, Lin YL, Liao KW, et al. miRTarBase update 2018: a resource for experimentally validated microRNAtarget interactions. Nucleic Acids Res. 2018;46:D296-302.

13. Wong $N$, Wang $X$. miRDB: an online resource for microRNA target prediction and functional annotations. Nucleic Acids Res. 2015;43:D146-52.

14. Park K, Kim KB. miRTar hunter: a prediction system for identifying human microRNA target sites. Mol Cell. 2013;35:195-201.

15. Zhang $\mathrm{S}$, Ding L, Li X, Fan $\mathrm{H}$. Identification of biomarkers associated with the recurrence of osteosarcoma using ceRNA regulatory network analysis. Int J Mol Med. 2019;43:1723-33.

16. Tian W, Jiang C, Huang Z, Xu D, Zheng S. Comprehensive analysis of dysregulated IncRNAs, miRNAs and mRNAs with associated ceRNA network in esophageal squamous cell carcinoma. Gene. 2019;696:206-18.

17. Bhan A, Soleimani M, Mandal SS. Long noncoding RNA and cancer: a new paradigm. Cancer Res. 2017;77:3965-81.

18. Slaby $\mathrm{O}$, Laga $\mathrm{R}$, Sedlacek $\mathrm{O}$. Therapeutic targeting of non-coding RNAs in cancer. Biochem J. 2017:474:4219-51.

19. Sun W, Yang Y, Xu C, Guo J. Regulatory mechanisms of long noncoding RNAs on gene expression in cancers. Cancer Gene Ther. 2017;216-217:105-10.

20. Su L, Wu A, Zhang W, Kong X. Silencing long non-coding RNA SNHG6 restrains proliferation, migration and invasion of Wilms' tumour cell lines by regulating miR-15a. Artif Cells Nanomed Biotechnol. 2019;47(1):2670-7.

21. Wang L, Liu D, Wu X, Zeng Y, Li L, Hou Y, et al. Long non-coding RNA (LnCRNA) RMST in triple-negative breast cancer (TNBC): expression analysis and biological roles research. J Cell Physiol. 2018;233:6603-12.

22. Zhang $X$, Rice $K$, Wang $Y$, Chen $W$, Zhong $Y$, Nakayama $Y$, et al. Maternally expressed gene 3 (MEG3) noncoding ribonucleic acid: isoform structure, expression, and functions. Endocrinology. 2010;151:939-47.

23. Lyu Y, Lou J, Yang Y, Feng J, Hao Y, Huang S, et al. Dysfunction of the WT1MEG3 signaling promotes AML leukemogenesis via p53-dependent and -independent pathways. Leukemia. 2017;31:2543-51.

24. Xie ZZ, Xiao ZC, Song YX, Li W, Tan GL. Long non-coding RNA Dleu2 affects proliferation, migration and invasion ability of laryngeal carcinoma cells through triggering miR-16-1 pathway. Eur Rev Med Pharmacol Sci. 2018;22:1963-70.

25. Rupaimoole R, Slack FJ. MicroRNA therapeutics: towards a new era for the management of cancer and other diseases. Nat Rev Drug Discov. 2017;16:203-22.

26. Guan H, You Z, Wang C, Fang F, Peng R, Mao L, et al. MicroRNA-200a suppresses prostate cancer progression through BRD4/AR signaling pathway. Cancer Med. 2019:8:1474-85.

27. Chen X, Liu K, Yang P, Kuang W, Huang H, Tu E, et al. microRNA-200a functions as a tumor suppressor by targeting FOXA1 in glioma. Exp Ther Med. 2019;17:221-9.

28. Zang Y, Tai Y, Wan B, Jia X. miR-200a-3p promotes the proliferation of human esophageal cancer cells by post-transcriptionally regulating cytoplasmic collapsin response mediator protein-1. Int J Mol Med. 2016:38:1558-64.

29. Wu Q, Lu RL, Li JX, Rong $\sqcup$. MiR-200a and miR-200b target PTEN to regulate the endometrial cancer cell growth in vitro. Asian Pac J Trop Med. 2017:10:498-502.

30. Tsouko E, Wang J, Frigo DE, Aydogdu E, Williams C. miR-200a inhibits migration of triple-negative breast cancer cells through direct repression of the EPHA2 oncogene. Carcinogenesis. 2015;36:1051-60.

31. Zhang M, Xue E, Shao W. Andrographolide promotes vincristine-induced SK-NEP-1 tumor cell death via PI3K-AKT-p53 signaling pathway. Drug Des Devel Ther. 2016;10:3143-52.

32. Zhao XS, Han B, Zhao JX, Tao N, Dong CY. MiR-155-5p affects Wilms' tumor cell proliferation and apoptosis via targeting CREB1. Eur Rev Med Pharmacol Sci. 2019:23:1030-7.

33. Li H, Huang D, Hang S. Salidroside inhibits the growth, migration and invasion of Wilms' tumor cells through down-regulation of miR-891b. Life Sci. 2019;222:60-8.

34. Theerakitthanakul K, Khrueathong J, Kruatong J, Graidist P, Raungrut P, Kayasut $\mathrm{K}$, et al. Senescence process in primary Wilms' tumor cell culture induced by p53 independent p21 expression. J Cancer. 2016;7:1867-76.

\section{Publisher's Note}

Springer Nature remains neutral with regard to jurisdictional claims in published maps and institutional affiliations. 